\title{
The Utilization of the Knee Exerciser Pro® R0M Device to Enhance Post-Operative Outcomes Following TKA
}

\author{
Tucker William F, Abbassi Ramzi and Connors Michael* \\ Knee Exerciser Pro, USA
}

Submission: August 01, 2017; Published: August 09, 2017

*Corresponding author: Connors Michael, Knee Exerciser Pro, USA, Website: www.KneeExerciser.com

\section{Introduction}

Total Knee Arthroplasty (TKA) is the most frequently performed orthopedic surgical procedure in the U.S. annually, with an estimated 700,000 procedures completed in 2016 [1]. The annual volume of TKAs is expected to grow to nearly 3.48 million by 2030. The goal of this procedure is to relieve pain, improve quality of life (QOL), and restore active knee flexion and extension Range of Motion (ROM). However, one of the most significant issues facing patients in their return to normal function is regaining full ROM, which can affect pain levels and QOL. Two significant contributing factors preventing patients from regaining full ROM are

a) An inability to effectively tolerate currentrecommended protocols for post-operative rehabilitation, including the use of a Continuous Passive Motion (CPM) machine and

b) Performing flexion and extension exercises unassisted while not under the direct supervision of a physical therapist.

Most protocols followed by orthopedic surgeons and hospitals for post-operative rehabilitation includes the use of a CPM machine in the first ten (10) to fourteen (14) days postoperatively. However, a literature review has shown that the CPM does not have clinically important effects on knee flexion ROM, pain, function or quality of life to justify its routine use [2]. It may reduce the risk of manipulation under anesthesia and risk of developing adverse events, however the quality of evidence supporting these findings are very low and low, respectively [2].

Although the intent of using a CPM is good, often times results achieved while using a CPM as part of an in-home rehabilitation program are less than desirable due to patient non-compliance or improper use. In the case of improper use, there is an increased risk of doing additional harm, which can lead to unnecessary complications and affect long term QOL. Additionally, insurance companies continue to push back on reimbursing for the use of CPM machines, further complicating the ability for orthopedic surgeons to recommend the use of this device as part of a post-operative rehabilitation program.

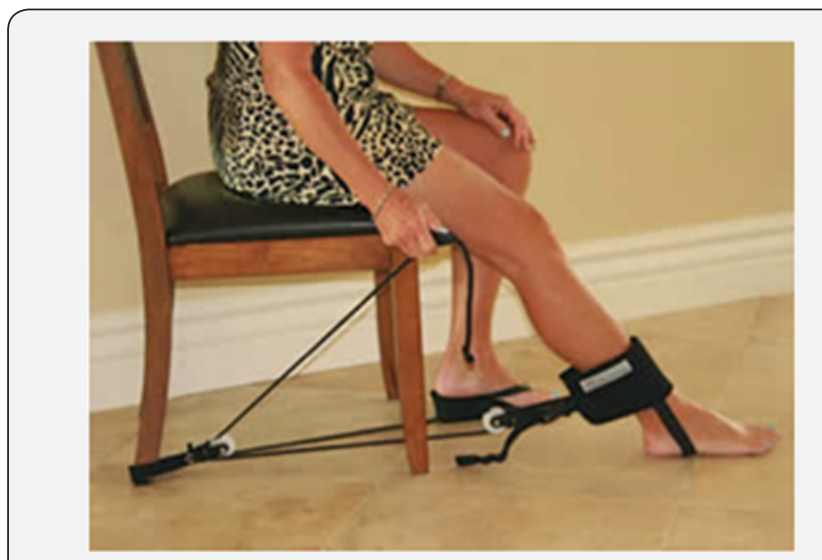

Figure 1A: Demonstrating Set-Up for Flexion.

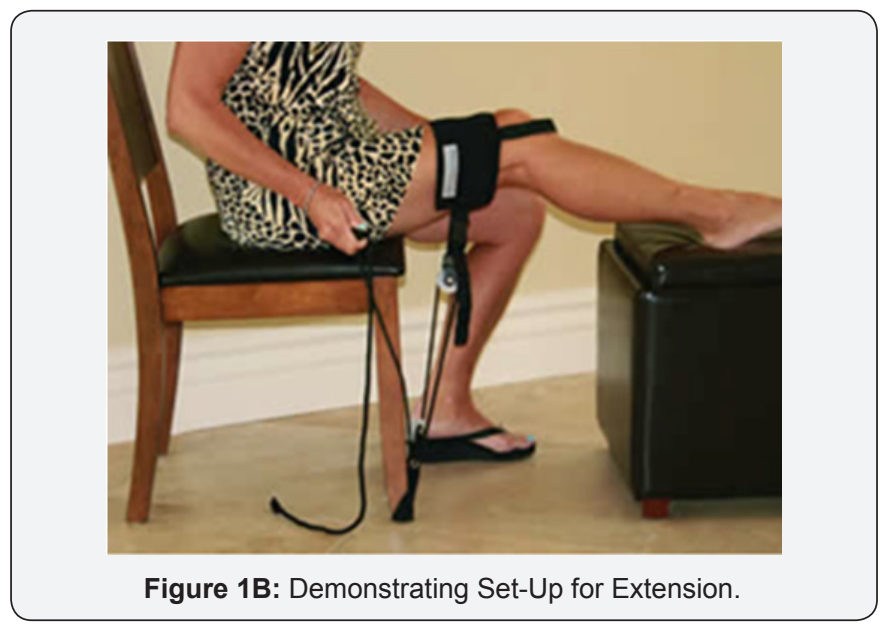

The Knee Exerciser Pro® was invented to specifically address the need to provide patients with a cost-effective method to safely perform flexion and extension exercises unassisted as 
part of a post-operative rehabilitation program (Figure 1A and 1B). The use of this device to drive passive ROM for knee flexion and extension is less likely to result in knee soreness post use. The device was specifically designed using a self-powered pulley system to multiply the amount of force created by a patient's own strength (Figure 1C). This pulley system more safely and effectively delivers improved ROM in flexion and extension.

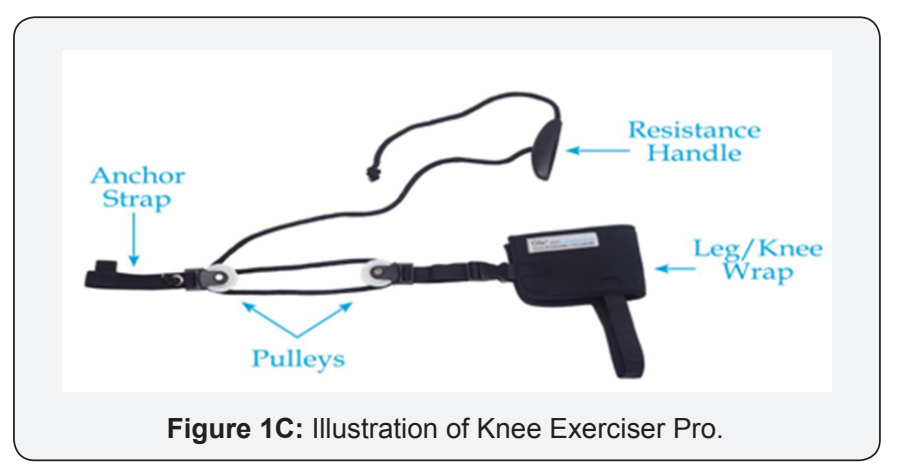

Methods

Fifteen (15) patients were recruited from a sample of convenience from Dr. William Tucker's Orthopedic practice in Dallas, Texas. Participants with a planned TKA were provided with an informed consent prior to surgery. Once a patient provided consent, the patient underwent an elective TKA by Dr. Tucker. Following discharge from the hospital, the patient was seen in the home setting for a total of 9 physical therapy visits over a three (3) week timeframe. This protocol, developed by Dr. Tucker, is an accelerated home physical therapy protocol as compared to the most common protocol, which ranges from twelve to eighteen (12-18) visits over a four to six (4-6) week timeframe. The most common home physical therapy protocol also involved daily exercises the patient was advised to complete on their own to enhance ROM and strength in the affected extremity.

At the baseline evaluation, a home health physical therapist assessed knee flexion ROM, knee extension ROM, baseline pain scales using the Numeric Pain Rating Scale, and fall risk using the Timed Up and Go (TUG) method. Knee flexion and extension were measured by the physical therapist using a goniometer, a common ROM measurement tool used in everyday practice. The Numeric Pain Rating Scale is a 0-10-point scale asking a patient to rate their current level of pain on a continuum from zero (0) meaning "no pain" to ten (10), meaning "unimaginable/ unspeakable". The TUG is a fall risk assessment that has been recognized in the literature as a valid, portable assessment to discern a patient's fall risk. The test involves standing from a seated position, walking 10 meters, and returning back to a seated position. A patient scoring more than 20 seconds represents a significant fall risk. The assessments were collected at baseline and again at discharge.

To provide a basis of comparison as a standard of care, a sample of ten (10) patients undergoing a TKA from Dr. William
Tucker's Orthopedic practice were provided a standard of care home therapy program, only the use of the Knee Exerciser Pro® device was not utilized in this sample. These patients only received a standard of care exercise program that consisted of range of motion and strengthening exercises for a total of nine (9) visits over three (3) weeks.

\section{Results}

This trial consisted of the use of the Knee Exerciser Pro $®$ device in a sample of fifteen (15) patients following a TKA seen for physical therapy in the home setting. The Knee Exerciser Pro ${ }^{\circledR}$ was utilized as an adjunctive tool to the standard of care physical therapy program. The variables assessed in this trial were baseline and discharge pain, ROM for knee flexion and extension and, fall risk using the TUG method. Data was analyzed for differences between means using independent paired samples t-test using SPSS 24.0 Statistical Analysis software.

The results of the analysis revealed multiple statistically significant conclusions. From these results, it is apparent that the difference in the mean from baseline to discharge for the four variables are all statistically significant: pain $(\mathrm{p}<0.0001)$, TUG ( $\mathrm{p}<0.001)$, knee flexion ROM ( $\mathrm{p}<0.0001)$, and knee extension ROM $(\mathrm{p}<0.017)$. These results indicate a significant improvement in multiple patient reported outcome measures when patients utilized the Knee Exerciser Pro in conjunction with typical home physical therapy program following TKA. Table 1 illustrates the means and standard deviations with corresponding $\mathrm{p}$-value from the t-test.

Table 1:

\begin{tabular}{|c|c|c|c|c|}
\hline \multicolumn{2}{|c|}{ Variable } & Mean & Std. Deviation & P-Value \\
\hline Pair 1 & $\begin{array}{c}\text { PainEVAL - } \\
\text { PainDC }\end{array}$ & 1.73333 & 1.38701 & 0.0001 \\
\hline Pair 2 & $\begin{array}{c}\text { TUGEVAL - } \\
\text { TUGDC }\end{array}$ & 17.06667 & 16.57652 & 0.0001 \\
\hline Pair 3 & $\begin{array}{c}\text { FLEXEVAL - } \\
\text { FLEXDC }\end{array}$ & -34.2 & 15.34927 & 0.0001 \\
\hline Pair 4 & $\begin{array}{c}\text { EXTEVAL - } \\
\text { EXTDC }\end{array}$ & -2.93333 & 4.18273 & 0.017 \\
\hline
\end{tabular}

Table 2:

\begin{tabular}{|c|c|c|c|c|}
\hline \multicolumn{2}{|c|}{ Variable } & Mean & Std. Deviation & P-Value \\
\hline Pair 1 & $\begin{array}{c}\text { PainEVAL - } \\
\text { PainDC }\end{array}$ & 2.75 & 1.83225 & 0.004 \\
\hline Pair 2 & $\begin{array}{c}\text { TUGEVAL - } \\
\text { TUGDC }\end{array}$ & 16.875 & 10.27393 & 0.002 \\
\hline Pair 3 & $\begin{array}{c}\text { FLEXEVAL - } \\
\text { FLEXDC }\end{array}$ & -36.125 & 16.60841 & 0.0001 \\
\hline Pair 4 & $\begin{array}{c}\text { EXTEVAL - } \\
\text { EXTDC }\end{array}$ & -5.125 & 2.29518 & 0.0001 \\
\hline
\end{tabular}

As compared to the Knee Exerciser Pro® group, the control group also experienced changes from baseline as noted in other trials analyzing a standard of care exercise program following 
a TKA. From these results, it is apparent that the difference in the mean from baseline to discharge for the four variables are all statistically significant: pain $(p<0.004)$, TUG $(p<0.002)$, knee flexion ROM $(\mathrm{p}<0.0001)$, and knee extension ROM $(\mathrm{p}<$ 0.001 ). These results indicate a significant improvement in multiple patient reported outcome measures when a traditional home physical therapy program is utilized following TKA. Table 2 illustrates the means and standard deviations with corresponding $\mathrm{p}$-value from the t-test.

In order to evaluate the effectiveness of the Knee Exerciser Pro ${ }^{\circledR}$ with standard of care versus standard of care alone, one needs to examine the mean and standard deviations of each variable to fully comprehend the differences between variables. While there are similarities between both groups, comparing means illustrates a more significant reduction in pain in the Knee Exerciser Pro® group, which indicates a more rapid reduction in pain using the device over standard of care alone. There was also a greater overall accelerated improvement in level of range of motion of flexion and extension at the beginning of the trial, with both groups demonstrating improvement over the three weeks but the Knee Exerciser Pro® group demonstrating a slight improvement over standard of care group.

\section{Limitations and Recommendations for Future Research}

No study would be complete without recognition of the potential limitations of this study as well as recommendations for future research assessing the treatment efficacy of the Knee Exerciser Proß. First, this study involved a sample of convenience from the orthopedic practice of a single physician. These results may not be generalizable to all patients of all Orthopedic Surgeons performing TKA all over the U.S. for a number of reasons, including differences in surgical technique and length of stay before discharge to home. Second, the sample size was small. In the treatment group 15 participants were enrolled, while in the control group we only included 10 participants. A power analysis of this study, with the power set at 0.8 and an effect size of 0.2 , yielded a need to enroll a total of 40 participants, 20 in each group. Since we only enrolled a total of 25 , a larger sample size may yield a more statistically significant difference between the two groups. Lastly, these results at present represent both a clinically significant difference as well as a slight statistically significant difference between the two groups.

There is also an acknowledgement and call for future studies examining the efficacy of this device on overall outcomes, as well as other variables not evaluated in this study. Additional study will likely strengthen the initial base of early evidence and provide additional supporting insight into the effectiveness of the device over the typical standard of care for a multitude of reasons. The principal investigators in this study suggest establishing a future study that examines a number of variables related to quality of life parameters. Second, a study exploring the potential impact on patient self-reported function may provide some insight into how post-operative function could be enhanced with the use of the Knee Exerciser Pro®. Third, a study examining the potential impact on reducing the overall cost of post-surgical therapy cost is highly recommended, as this small sample size has indicated that fewer visits paired with use of the Knee Exerciser Pro $₫$ generated improved outcomes over the current standard of care. Lastly, a study that is focused on further examining a more comprehensive pain and fall risk assessment might shed light on how the use of the device may more comprehensively positively impact the use of opioid analgesic medications as well as decrease risk for falls in a population of patients following TKA.

\section{Conclusion}

From the results of this clinical trial, there is statistically significant evidence to illustrate that the Knee Exerciser Pro®, when utilized as an adjunctive intervention to a home physical therapy program, can help enhance knee ROM, decrease pain, and decrease fall risk, as compared to a standard of care home exercise alone group. The use of this device in this trial assisted in successfully facilitating a decrease in the total cost of care for the episode by only requiring nine (9) home health physical visits, as compared to the typical twelve to eighteen (12-18) most patients are provided following TKA. The direct decrease in cost of care delivery and diminished length of time of the home health episode is a substantial factor to consider, given that the volume of TKA procedures is set to exceed 3.48 million per year by 2030 [3].

There were no adverse events reported during this trial, with no increase in patient soreness attributed to the Knee Exerciser Pro®. This lack of soreness with device use was likely a result in a higher degree of patient compliance and engagement, which lead to the best possible outcomes with the least likely risk for adverse events. In addition, the fall risk for patients following TKA is of significant concern. Although the use of opioids for pain is decreasing due to concerns over addiction and anesthesia techniques are becoming more sophisticated, this patient population is still considered to have an increased fall risk, which can result in further injuries and complications. The substantial decrease in pain while using the Knee Exerciser Pro® ${ }^{\circledR}$ will likely lead to a decrease in reliance on pain medications which, further decreases the risk for medication addiction.

The results of this trial suggest that the Knee Exerciser Pro®, when used as an accompaniment to a standardized inhome physical therapy protocol, improves knee flexion and extension ROM, decreases pain scores and improves TUG. The improvement in patient QOL, the potential cost savings realized for the episode of care due to fewer visits needed and the decrease in fall risk are additional direct benefits of utilizing Knee Exerciser Pro $®$ as part of a post-operative rehabilitation program. 


\section{References}

1. Arora AS, Chung KC, Otto W (2006) Madelung and the Recognition of Madelung's Deformity. J Hand Surg 31(2): 177-182.

2. Kozin SH, Zlotolow DA (2015) Madelung Deformity. J Hand Surg Am 40(10): 2090-2098.

3. Huguet S (2014) Radiological and clinical analysis of Madelung's deformity in children. Orthopedics \& Traumatology: Surgery \& Research 100: 349-352.

4. Ghatan AC, Hanel DP (2013) Madelung deformity. J Am AcadOrthop Surg 21: 372-382.
5. Tranmer A, Laub D (2016) Madelung deformity. Interesting Case.

6. McCarroll HR, James MA, Newemeyer WL, Manske PR (2010) Madelung's deformity: Diagnostic thresholds of radiographic measurements. J Hand Surg Am 35: 807-812.

7. Ly-Pen D, Andreu JL (2014) Madelung's deformity. ReumatolClin 10(2): 125-126.

8. Brooks TJ (2001) Madelung deformity in a collegiate gymnast: a case report. Journal of Athletic Training 36(2): 170-173.

\section{Your next submission with Juniper Publishers will reach you the below assets}

- Quality Editorial service

- Swift Peer Review

- Reprints availability

- E-prints Service

- Manuscript Podcast for convenient understanding

- Global attainment for your research

- Manuscript accessibility in different formats

( Pdf, E-pub, Full Text, Audio)

- Unceasing customer service

Track the below URL for one-step submission https://juniperpublishers.com/online-submission.php 